Dorota Wyrostkiewicz', Monika Szturmowicz', Iwona Bartoszuk', Izabela Siemion-Szcześniak', Lilia Jakubowska' ${ }^{2}$, Ewa Augustynowicz-Kopeć ${ }^{3}$, Jan Kuś ${ }^{1}$

${ }^{1} I^{\text {st }}$ Department of Lung Diseases National Institute of Tuberculosis and Lung Diseases, Warsaw, Poland

${ }^{2}$ Department of Radiology National Institute of Tuberculosis and Lung Diseases, Warsaw, Poland

${ }^{3}$ Department of Microbiology National Institute of Tuberculosis and Lung Diseases, Warsaw, Poland

\title{
Nontuberculous mycobacterial lung disease in a patient with COPD and bronchiectasis, with radiological signs of lung tumor
}

The study was carried out within the statutory activity of the National Research Institute of Tuberculosis and Lung Diseases (topic 1.3)

\begin{abstract}
Mycobacterial lung disease is caused by nontuberculous mycobacteria (NTM), also known as atypical mycobacteria. NTM are widely distributed in the environment, particularly in soil and water; they may colonize the airways, gastrointestinal tract and genitourinary system, without the apparent signs of disease. Nevertheless, in some risk groups such as patients with chronic lung diseases or with immunodeficiency, mycobacterial lung disease is identified. Recently, increased recognition of mycobacterial lung disease in chronic obstructive pulmonary disease (COPD) patients has been observed, especially in those treated with high doses of inhaled corticosteroids.

In the present paper, we describe the patient treated for many years due to COPD and bronchiectasis, with clinical and radiological picture suggestive of lung tumor, in whom final diagnosis of mycobacterial lung disease caused by Mycobacterium avium was made.

Key words: nontuberculous mycobacterial lung disease, nontuberculous mycobacteria, Mycobacterium avium, bronchiectasis, lung tumor
\end{abstract}

Adv Respir Med. 2018; 86: 17-22

\section{Introduction}

Mycobacteriosis is a group of diseases caused by nontuberculous mycobacteria, also called atypical, that includes species other than $\mathrm{Myco-}$ bacterium tuberculosis. The English abbreviations of these bacteria such as NMT (nontuberculous mycobacteria) and MOTT (mycobacteria other than tuberculosis) are also used. Nontuberculous mycobacteria are widely spread in the environment, mostly in soil and water tanks, as well as in pipelines. For humans, these bacteria are mostly just saprophytes, they may colonize the airways, gastrointestinal tract and genitourinary tract [1-3]. Patients at higher risk of mycobacteriosis are those with chronic lung diseases (such as bronchiectasis, cystic fibrosis, chronic obstructi- ve pulmonary disease (COPD), pneumoconiosis, pulmonary alveolar proteinosis, past pulmonary tuberculosis), diabetes, renal failure, pulmonary hypertension and the impaired immune system (such as individuals infected with human immunodeficiency virus (HIV), following bone marrow/ /organ transplantation, or receiving immunosuppressants) [4-7].

The NTM infections most likely occur as a result of contact with bacteria in the environment, for example inhalation of contaminated water spray. To date, there is no evidence that the infection may be transferred between humans and from animals to humans, however, there are single reports that such transmission of $M$. abscessus may have been possible between patients with cystic fibrosis [7].

Address for correspondence: Dorota Wyrostkiewicz, Ist Dept of Lung Diseases National Institute of Tuberculosis and Lung Diseases, ul. Plocka 26, 01-138 Warsaw, e-mail: d.wyrostkiewicz@igichp.edu.pl

DOI: 10.5603/ARM.2018.0005

Received: 14.11.2017

Copyright (C) 2018 PTChP

ISSN 2451-4934 
COPD is characterized by a persistent limitation of airflow through the lower respiratory tract, which usually progresses and is caused by excessive inflammation of the bronchi and lungs in response to adverse reaction to gases and dust. In Poland, COPD predominantly originates from smoking tobacco. Exacerbations and concomitant diseases affect the course of COPD in individual patients [8]. Chronic inflammation in people with COPD leads to changes in the structure and functioning of the respiratory system, in some of the patients bronchiectasis are also diagnosed. COPD as well as bronchiectasis constitute risk factors for mycobacteriosis, and therefore, the disease should be considered in the course of diagnostics of newly discovered radiological changes in the lungs [9-12].

The present paper describes a case report of pulmonary mycobacteriosis mimicking neoplastic lung disease in a patient with COPD and bronchiectasis.

\section{Case report}

A 63-year-old smoking patient, with COPD (group B), advanced bronchiectasis and a history of tuberculosis in childhood was admitted to the hospital due to a suspected tumor located in the right lung. The patient has been complaining of increased cough with purulent sputum expectoration, minor hemoptysis, dyspnea on exertion, low-grade fever and weight loss of $5 \mathrm{~kg}$ within past 6 months. The patient has been treated for several years with inhaled drugs such as beta-agonist, cholinolytic and corticosteroid (budesonide $800 \mathrm{mcg} /$ day). A month before the present hospitalization, he was admitted to another hospital due to an infectious exacerbation of COPD and received amoxicillin/clavulanic acid, ciprofloxacin and doxycycline with clinical improvement.

A chest CT scan prior to hospitalization revealed a solid lesion in the segment 6 of the right lung measuring approximately $5 \mathrm{~cm}$. The lesion enhanced with contrast. Furthermore, the scan showed a layer of fluid in the right pleural cavity measuring up to $17 \mathrm{~mm}$ and enlarged lymph nodes in the right hilum up to $15 \mathrm{~mm}$. Laboratory tests on admission did not reveal an increase in inflammatory markers. Capillary gasometry disclosed hypoxemia $\left(\mathrm{pO}_{2} 58 \mathrm{~mm} \mathrm{Hg}\right)$ with hypocapnia $\left(\mathrm{pCO}_{2} 34 \mathrm{~mm} \mathrm{Hg}\right)$, and oxygen saturation amounting to $92 \%$. Spirometry revealed features of severe bronchial obstruction, with $\mathrm{FEV}_{1}$ amounting to $41 \%$ of the predicted value. X-Ray of the chest revealed signs of chronic bronchitis
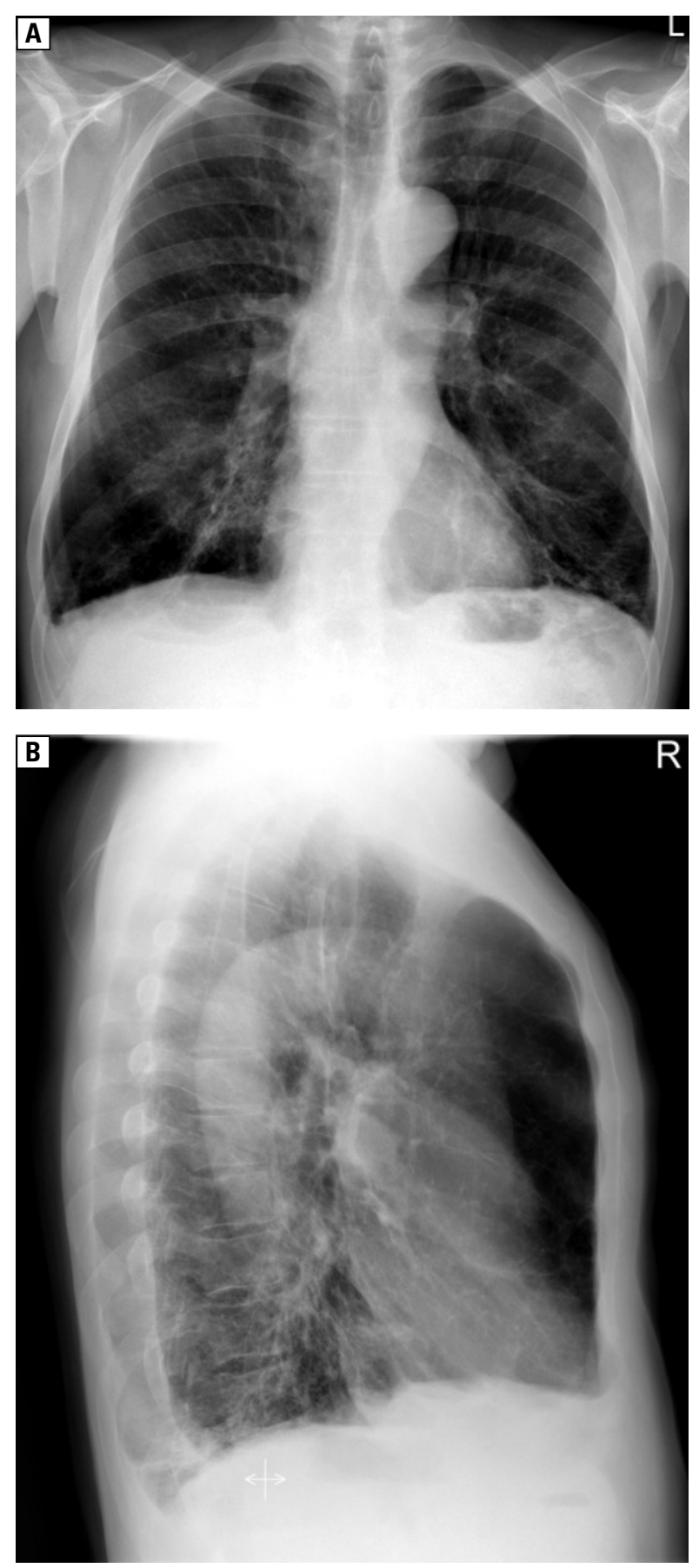

Figure 1A, B. Frontal and lateral chest radiographs. Emphysema. Bronchial wall thickening in the lower lobes. Blunting of the right costophrenic angle suggesting pleural effusion

and bronchiectasis with possible trace of fluid in the right pleural cavity (Fig. 1). A focal lesion described in the previous CT scan was not visible on the X-ray. Thus chest CT scan was repeated and a partial regression of the focal lesion in the segment 6 of the right lung was observed. At that time the lesion measured $17 \times 32 \times 20 \mathrm{~mm}$ and presented with an irregular silhouette. There was also a small trace of fluid in the right pleural 


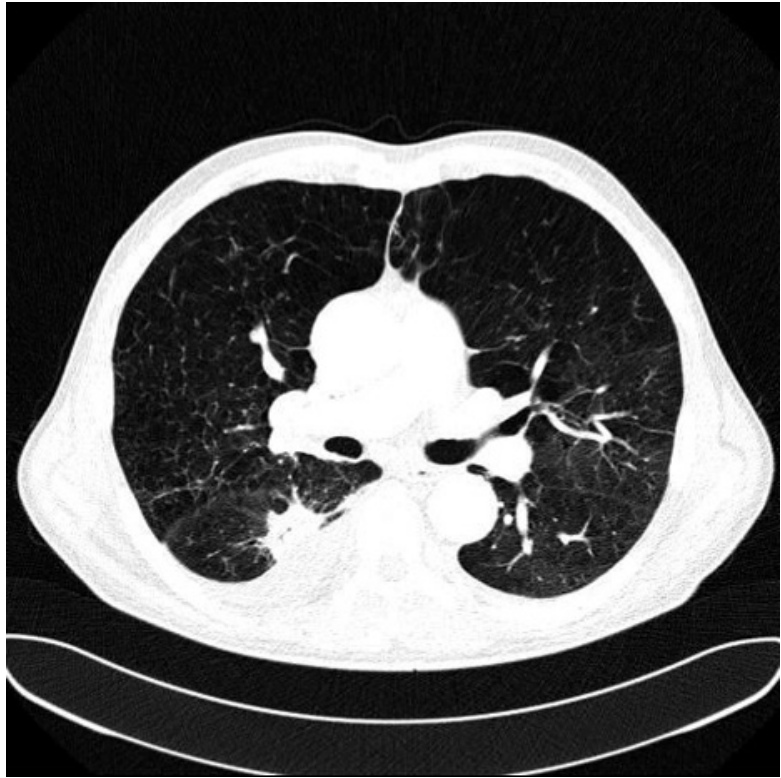

Figure 2. Axial CT image (lung window) shows a spiculated nodule in the sixth segment of right lower lobe mimicking primary lung cancer. Right-sided pleural effusion. Emphysema

cavity, the thickness of fluid layer measured up to $9 \mathrm{~mm}$ (Fig. 2). Despite the partial regression of the focal lung lesion, in the differential diagnosis, we took into account the neoplastic disease with the coexisting inflammatory reaction, that was diminished due to antibiotics use.

Bronchoscopy was performed, and bronchial secretions were sampled for examination. Both bronchi presented with macroscopic intensive inflammation and scarring resulting from nodal perforations. Bronchial washings bacterioscopy targeted on acid-resistant mycobacteria and genetic testing for M. tuberculosis complex were negative. The culture for mycobacterium was still under observation. Cytology of the bronchial secretion revealed only inflammatory cells. The IGRA test (Qantiferon Tb Gold) was negative.

Considering the entire clinical presentation, past tuberculosis in childhood and the chest CT results (characteristic location of the lesion), despite a negative result of the direct and genetic testing, the patient was diagnosed with tuberculosis and antituberculosis treatment was introduced. The patient received rifampicin (RMP), isoniazid (INH), and pyrazinamide (PZA). After ophthalmologist consultation, etambutol (EMB) was also administered. The patient was discharged with recommendation to continue anti-tuberculous treatment until the results of the culture were obtained. After 4 weeks of incubation of bronchial discharge on liquid medium, the growth of MOTT was observed. The bacteria

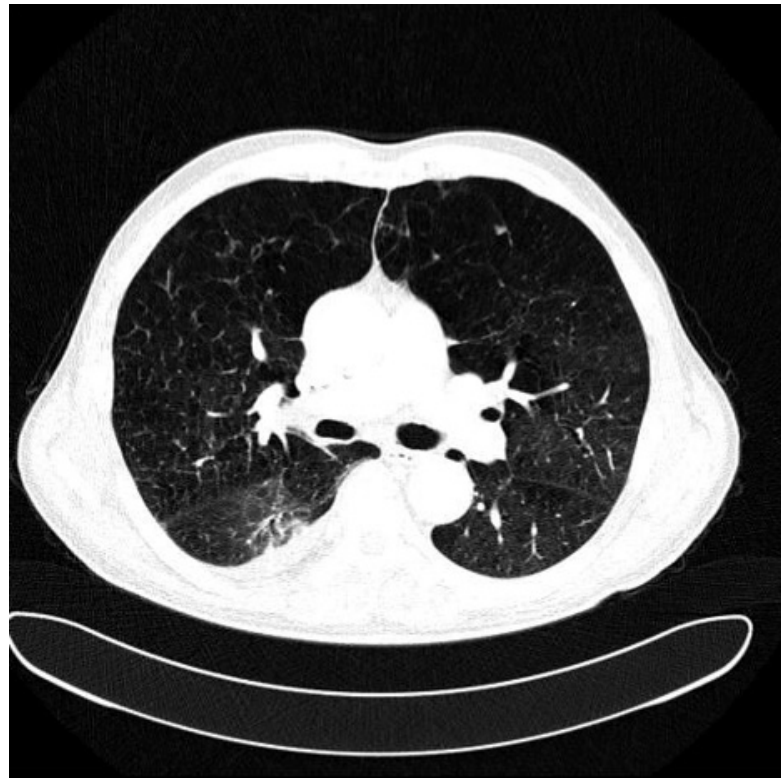

Figure 3. CT scan shows nearly complete regression of nodule in the right lower lobe and pleural effusion after three months

were then identified using molecular method as $M$. avium. Due to good clinical response and the patient's acceptance, the treatment with RMP, INH and EMB was continued and PZA was stopped. A follow-up chest CT scan performed 3 months later revealed a significant regression of disease, the focal lesion and the pleural fluid were not visible (Fig. 3). After 4 months of treatment, sputum cultures became negative. According to the guidelines [1,2], the treatment was continued in an outpatient setting for 12 months from the moment of mycobacteria eradication.

\section{Discussion}

The prevalence of pulmonary mycobacteriosis is still increasing. In the United States, it is currently higher than prevalence of tuberculosis [7].

The increased prevalence is also influenced by the progress in microbiological diagnostics and widespread use of high-resolution computed tomography capable of detecting lung lesions that are not visible on chest X-rays. In industrialized countries, this increase in morbidity may also be related to aging of population [13].

Patients with COPD are at risk of mycobacteriosis development, especially those with coexisting bronchiectasis [13].

Among 126 subjects with COPD who underwent surgical lung-volume reduction in two British hospitals, signs of NTM infections were detected in $10 \%$ of postsurgical histological ma- 
terial [14]. Basing on population studies carried out in Denmark, the authors concluded that in patients with COPD, the risk of mycobacterial infection is 15 times higher, and in individuals with bronchiectasis - 187 times higher in comparison with the general population [7]. In every group, the risk increased significantly when the subjects received corticosteroids, and was dose -dependent [9, 11, 12].

The presented patient had three potential risk factors of mycobacteriosis, namely COPD, bronchiectasis, and chronic inhalation of corticosteroid.

Huang et al. [15] demonstrated that possible isolation of nontuberculous mycobacteria in patients with COPD correlates with the number of exacerbations. In their group of patients, similarly to our case, MAC (Mycobacterium avium complex) was the most common diagnosis. Regardless of the predisposing factors, in many parts of the world, there is an increase in the incidence of MAC infection. In a study based on the data collected across the globe, MAC was found to be most common in Australia and North America, whereas in Europe, $M$. xenopii was the most frequently isolated strain (more often in southern Europe) [16].

Due to the high prevalence of mycobacteria in the human environment, proper interpretation of the culture result is crucial. Nontuberculous mycobacteria bacteria are relatively unaggressive, and therefore, it is necessary to differentiate between the disease and colonization. Mycobacteriosis is diagnosed in a patient fulfilling clinical, radiological and microbiological criteria elaborated by the American Thoracic Society (ATS) and Infectious Diseases Society of America (IDSA) (Table 1) [1].

The clinical symptoms of mycobacteriosis in patients with chronic lung disease are masked by the symptoms of the underlying lung disease.
Therefore, it is important to focus on new and chronic symptoms such as: weakness, fatigue, fever, dyspnea, hemoptysis, chest pain, slimming, and night sweats, which may indicate mycobacterial infection [3]. In the case described herein, the patient had experienced low-grade fever for several months as well as increased expectoration of purulent sputum, sometimes with trace of blood and weight loss.

Radiologic manifestations of pulmonary mycobacteriosis include infiltrative and fibrotic-like lesions (similar to tuberculosis) or nodules and bronchiectasis. The most common radiological presentation of mycobacteriosis caused by MAC includes small nodules and bronchiectasis [17]. Studies carried out by Haiqing Chu et al. [18] revealed that MAC was detected more frequently than $M$. abscessus and M. kansasii in patients with bronchiectasis, whereas patients without bronchiectasis were mostly diagnosed with M. kansasii and M. xenopii.

Single infiltrative lung lesion described on chest CT in the presented patient, was not typical of mycobacteriosis and suggested rather neoplastic disease, especially, in the person with a long history of cigarette smoking. As partial regression of the above described lesion was observed on a second chest CT, performed in our department after the treatment with antibiotics in the previous hospital, we took into account the neoplastic disease with coexisting inflammatory pathology. However, lack of neoplastic infiltration in bronchoscopy, negative results of cytological examination of the sputum and bronchial secretions, as well as almost complete regression of chest CT pathology observed 3 months later, allowed for the exclusion of malignancy in the presented patient.

On the basis of the anamnesis, location of the lesion (segment 6) and bronchoscopic presenta-

\section{Table 1. Diagnosis of nontuberculous mycobacteria lung disese [1]}

\section{Clinical criteria}

1. Pulmonary symptoms, nodular or cavitary opacities on chest radiograph, or an HRCT scan that shows multifocal bronchiectasis with multiple small nodules and

2. Appropriate exclusion of other diagnoses

\section{Microbiologic criteria}

1. Positive culture results from at least two separate expectorated sputum samples. (If the results from the initial sputum samples are nondiagnostic, consider repeat sputum AFB smears and cultures) or

2. Positive culture results from at least one bronchial wash or lavage or

3. Transbronchial or other lung biopsy with mycobacterial histopathologic features (granulomatous inflammation or AFB) and positive culture for NTM or biopsy showing mycobacterial histopathologic features (granulomatous inflammation or AFB) and one or more sputum or bronchial washings that are culture positive for NTM

HRCT — high resolution computed tomography; AFB — acid fast bacilli; NTM — nontuberculous mycobacteria 
tion, despite negative results of direct sputum testing for mycobacteria and negative results of genetic test, the patient was diagnosed with tuberculosis, and standard TB treatment was administered. The diagnosis of NTMLD was made when the sputum culture revealed the growth of M. avium, 4 weeks later.

Bacteriological diagnostics of mycobacteriosis is mainly based on bacterioscopy and sputum, bronchial discharge or tissue sample cultures. Individual species of mycobacteria differ in terms of pathogenicity. The positive culture with the growth of $M$. kansasii or $M$. malmoense results in clinical manifestation in over $70 \%$ of cases. M. szulgai also presents with high pathogenicity. Mycobacterium avium complex, M. xenopii and $M$. abscessus manifest intermediate pathogenicity. The other mycobacteria are less pathogenic [17].

Genetic probe is an important diagnostic tool that detects the genome of $M$. tuberculosis complex. Genetic testing is particularly indicated in patients with positive result of bacterioscopy, when it allows to differentiate quickly between tuberculosis and mycobacteriosis.

In the case we describe, the genetic test was negative, and therefore, it did not have any clinical significance. A positive result may have supported the diagnosis of tuberculosis.

Tuberculin skin testing (TST) and IGRA testing are not very useful in the differential diagnosis of mycobacteriosis and active tuberculosis. The TST test may be positive in NTM disease, because $M$. tuberculosis and all NTM have the same antigens as the entire strain of mycobacterium. Patients with NTM infections usually have negative IGRA test results. M. kansasii, M. marinum and M. shulgai are the exceptions, since they belong to a few NTMs, which - similarly to the $M$. tuberculosis - produce ESAT-6 and CFP-10 proteins used as antigens in IGRA tests [19]. The negative result of IGRA in the presented patient did not rule out active tuberculosis and did not have any clinical utility in case of mycobacteriosis. Only microbiological examinations are important in differentiation between mycobacterial infection and tuberculosis.

Treatment of patients with mycobacteriosis is difficult. In most cases multidrug regimes are used according to the guidelines of ATS and BTS $[1,2]$. Due to long duration of treatment (12 months since negative culture result) and possible adverse effects of administered drugs, the risk and benefits of treatment should be evaluated individually for each patient.
In the case we describe, despite the low pathogenicity of $M$. avium, it was decided to continue the therapy, due to the symptomatic course of the disease and good treatment tolerability.

In MAC infections, the routine treatment includes macrolide and etambutol with rifampicin. In severe cases, aminoglycoside should be added. Sometimes patients undergo surgery. The treatment results have significantly improved after implementation of new macrolides such as clarithromycin and azithromycin [20].

The patient described in our case study initially received RMP, INH, EMB and PZA because tuberculosis was suspected. After having received a positive culture for M. avium, pyrazinamide was discontinued, and the other drugs were maintained.

Treatment of mycobacteriosis requires strict control in the outpatient settings, as it is a longterm process. In addition, serial direct sputum examinations and cultures are necessary. Clinical improvement and negative culture should be obtained after 3-6 months of treatment $[1,2]$. The therapy should be continued for one year after the negative culture result $[1,2]$.

In the case we describe, the patient responded well to treatment. After 4 months almost complete regression of radiological features of disease and negativization of sputum cultures were observed, thus the previously administered treatment was continued for the next 12 months.

Mycobacteriosis should be always considered in all patients with risk factors who are hospitalized due to new clinical symptoms and radiological characteristics in the lungs. Diagnosis of mycobacteriosis in the course of chronic lung condition may be especially difficult, and therefore, doctors should be aware of this possibility.

\section{Conflict of interest}

The authors declare no conflict of interest.

\section{References:}

1. Griffith DE, Aksamit T, Brown-Elliott BA, et al. ATS Mycobacterial Diseases Subcommittee, American Thoracic Society, Infectious Disease Society of America. An official ATS/IDSA statement: diagnosis, treatment, and prevention of nontuberculous mycobacterial diseases. Am J Respir Crit Care Med. 2007; 175(4): 367-416, doi: 10.1164/rccm.200604-571ST, indexed in Pubmed: 17277290.

2. Haworth C, Banks J, Capstick T, et al. British Thoracic Society Guideline for the management of non-tuberculous mycobacterial pulmonary disease (NTM-PD). BMJ Open Respiratory Research. 2017; 4(1): e000242, doi: 10.1136/bmjresp-2017-000242.

3. Wilińska E. Szturmowicz Mykobakteriozy płuc - obraz kliniczny, diagnostyka i leczenie. Pneumonol Alergol Pol. 2010; 78(2): 138-147.

4. Hughes WT. Mycobacterial infections in bone marrow transplant recipients. Biol Blood Marrow Transplant. 2000; 6(4): 359-360, indexed in Pubmed: 10917570. 
5. Wallace RJ, Zhang Y, Brown BA, et al. Polyclonal Mycobacterium avium complex infections in patients with nodular bronchiectasis. Am J Respir Crit Care Med. 1998; 158(4): 1235-1244, doi 10.1164/ajrccm.158.4.9712098, indexed in Pubmed: 9769287.

6. Kubo K, Yamazaki Y, Hachiya T, et al. Mycobacterium avium -intracellulare pulmonary infection in patients without known predisposing lung disease. Lung. 1998; 176(6): 381-391, indexed in Pubmed: 9780296.

7. Rowińska-Zakrzewska E. Mikobakterioza płuc. Rzadka choroba czy narastający problem epidemiologiczny? Pneumonologia i Alergologia Polska. 2014; 82(6): 486-488, doi: 10.5603/ piap.2014.0064.

8. Śliwiński P, Górecka D, Jassem E, et al. Zalecenia Polskiego Towarzystwa Chorób Płuc dotyczące rozpoznawania i leczenia przewlekłej obturacyjnej choroby płuc. Pneumonologia i Alergologia Polska. 2014; 82(3): 227-263, doi: 10.5603/ piap.2014.0030.

9. Andréjak C, Nielsen R, Thomsen VØ, et al. Chronic respiratory disease, inhaled corticosteroids and risk of non-tuberculous mycobacteriosis. Thorax. 2013; 68(3): 256-262, doi: 10.1136/ thoraxjnl-2012-201772, indexed in Pubmed: 22781123.

10. Andréjak C, Thomsen VØ, Johansen IS, et al. Nontuberculous pulmonary mycobacteriosis in Denmark: incidence and prognostic factors. Am J Respir Crit Care Med. 2010; 181(5): 514-521, doi: 10.1164/rccm.200905-0778OC, indexed in Pubmed: 20007929.

11. Hojo M, Iikura M, Hirano S, et al. Increased risk of nontuberculous mycobacterial infection in asthmatic patients using long-term inhaled corticosteroid therapy. Respirology. 2012; 17(1): 185-190, doi: 10.1111/j.1440-1843.2011.02076.x, indexed in Pubmed: 21995339.

12. Brode SK, Campitelli MA, Kwong JC, et al. The risk of mycobacterial infections associated with inhaled corticosteroid use. Eur Respir J. 2017; 50(3), doi: 10.1183/13993003.00037-2017, indexed in Pubmed: 28931659.
13. Gatheral T, Kumar N, Sansom B, et al. COPD-related bronchiectasis; independent impact on disease course and outcomes. COPD. 2014; 11(6): 605-614, doi: 10.3109/15412555.2014.922174, indexed in Pubmed 24983298

14. Char A, Hopkinson NS, Hansell DM, et al. Evidence of mycobacterial disease in COPD patients with lung volume reduction surgery; the importance of histological assessment of specimens: a cohort study. BMC Pulm Med. 2014; 14: 124, doi 10.1186/1471-2466-14-124, indexed in Pubmed: 25086862.

15. Huang CT, Tsai YJ, Wu HD, et al. Impact of non-tuberculous mycobacteria on pulmonary function decline in chronic obstructive pulmonary disease. Int J Tuberc Lung Dis. 2012; 16(4): 539-545, doi: 10.5588/ijtld.11.0412, indexed in Pubmed: 22325332.

16. Prevots DR, Loddenkemper R, Sotgiu G, et al. Nontuberculous mycobacterial pulmonary disease: an increasing burden with substantial costs. Eur Respir J. 2017; 49(4), doi: 10.1183/13993003.00374-2017, indexed in Pubmed: 28446563.

17. Korzeniewska-Koseła M. Mykobakteriozy: występowanie, postaci, rozpoznawanie i leczenie. http://www.mp.pl/pulmonologia/artykuly-wytyczne/ pogladowe/show.html?id=116396

18. Chu H, Zhao L, Xiao H, et al. Prevalence of nontuberculous mycobacteria in patients with bronchiectasis: a meta-analysis. Arch Med Sci. 2014; 10(4): 661-668, doi: 10.5114/ aoms.2014.44857, indexed in Pubmed: 25276148.

19. Sato R, Nagai H, Matsui $H$, et al. Interferon-gamma release assays in patients with Mycobacterium kansasii pulmonary infection: A retrospective survey. J Infect. 2016; 72(6): 706-712, doi: 10.1016/j.jinf.2016.03.011, indexed in Pubmed: 27025204.

20. Griffith DE, Brown BA, Girard WM, et al. Azithromycin activity against Mycobacterium avium complex lung disease in patients who were not infected with human immunodeficiency virus. Clin Infect Dis. 1996; 23(5): 983-989, indexed in Pubmed: 8922790. 